International Journal of Pure and Applied Mathematics

Volume 107 No. $4 \quad 2016,939-948$

ISSN: 1311-8080 (printed version); ISSN: 1314-3395 (on-line version)

url: http://www.ijpam.eu

doi: 10.12732/ijpam.v107i4.11



\title{
A RECURRENCE RELATION WITH COMBINATORIAL IDENTITIES
}

\author{
George Grossman ${ }^{1}$, Aklilu Zeleke ${ }^{2}$, Xinyun Zhu ${ }^{3}$, Tomas Zdrahal ${ }^{4}$ \\ ${ }^{1}$ Department of Mathematics \\ Central Michigan University \\ Mount Pleasant, MI 48859, USA \\ ${ }^{2}$ Lyman Briggs College and Department of Statistics and Probability \\ Michigan State University \\ East Lansing, MI 48824, USA \\ ${ }^{3}$ Department of Mathematics \\ University of Texas of the Permian Basin \\ Odessa, TX 79762, USA \\ ${ }^{4}$ Katedra matematiky \\ Palacký University \\ Olomouc, CZECH REPUBLIC
}

\begin{abstract}
In the present paper, we consider a pair of recurrence relations whose simultaneous solution involves two parameters $k, n$. We also find generating function of the sequence. Identities related to the Fibonacci and Lucas numbers are given.
\end{abstract}

AMS Subject Classification: 65Q30, 05A19, 11B39

Key Words: recurrence relations, combinatorial identities, Fibonacci and Lucas numbers

\section{Introduction}

The study of linear recurrence relations has a long history. Theon of Smyrna (130 AD) studied a coupled pair of linear recurrence relations to approximate $\sqrt{2}$, [2]. Riordan [10] gave a summary of methods to investigate and find combinatorial identities. For a comprehensive catalogue of combinatorial and

Received: September 25, 2015

Published: May 5, 2016

$\S$ Correspondence author
(C) 2016 Academic Publications, Ltd. url: www.acadpubl.eu 
other results see Gould [3]. One can also find well-known combinatorial identities involving the Fibonacci and Lucas numbers in Koshy [8]. The following result, G. Pólya and G. Szegö [9 ], (pp. 6, No.39),

$$
\sum_{k=0}^{n}(-1)^{n-k} 2^{2 k}\left(\begin{array}{c}
n+k+1 \\
2 k+1
\end{array}\right)=n+1
$$

is significant because of its derivation. From historical perspective the derivation of (1.1) follows from finding the coefficient of $x^{2 n+1}$ in

$$
\sum_{k=0}^{n} \frac{1}{2}(1+2 x)^{n+k+1}\left(-x^{2}\right)^{n-k} .
$$

From [9 ], pp. 159 (or by application of MAPLE software,) (1.2) simplifies to,

$$
\frac{1}{2}(1+2 x)^{n+1}\left((1+2 x)^{n+1}-\left(-x^{2}\right)^{n+1}\right)(1+x)^{-2} .
$$

By inspection, in (1.3), it suffices to consider the term $\frac{1}{2}(1+2 x)^{2 n+2}(1+x)^{-2}$, which, upon division, has remainder $(1-(2 n+2)(2 x+2)) / 2$. Thus, the coefficient of $\frac{1}{2}(1+2 x)^{2 n+2}(1+x)^{-2}$ is $n+1$.

This nontrivial derivation depends on several analytical insights. Riordan [10] has also shown that (1.1) satisfies that recurrence relation

$$
f_{n}=2 f_{n-1}-f_{n-2}, f_{0}=1, f_{1}=2 .
$$

Thus, one can see how ideas from analysis, combinatorics and recurrence relations are used to derive (1.1).

The objective of this paper is to investigate a pair of (nonhomogeneous) second order recurrence relations in one unknown $w_{k, n}$ involving two subscripts $n$ and $k$. We then outline a methodology to derive new representations of Fibonacci numbers and give two results. Recurrence relations and generating functions are essentially the focus of the present paper. The paper is organized as follows. In Section 2 we find the generating function for $w_{k, n}$. Several other formulas for $w_{k, n}$ are given, including the general solution using two approaches. In Section 3 we will present some representations of the Fibonacci numbers by applying the main results. 


\section{Main Results}

The generating function of the standard linear recurrence relation

$$
-w_{n}-w_{n+1}+w_{n+2}=0
$$

that generates the Fibonacci numbers with $F_{0}=0, F_{1}=1$, is known to be

$$
f(x)=\frac{1}{1-x-x^{2}}
$$

Consider now the linear recurrence relation with initial conditions

$$
-w_{n}-b w_{n+1}+c w_{n+2}=0
$$

with initial conditions $w_{0}=1 / c, w_{1}=1 / c+b / c^{2}$. Then, by using standard methods [11], [4], one can show that its generating function has the form

$$
f(x)=\frac{1+x}{c-b x-x^{2}} .
$$

Similarly the generating function of the nonhomogeneous linear recurrence equation

$$
\begin{gathered}
-w_{n}-b w_{n+1}+c w_{n+2}=1, w_{0}=1 / c, w_{1}=1 / c+b / c^{2} \text { is } \\
f(x)=\frac{1}{\left(c-b x-x^{2}\right)(1-x)} .
\end{gathered}
$$

The following theorems show how to generalize this result for a double indexed recurrence relation.

Theorem 1. Let $w_{0,0}=1 / c$ and $w_{0,1}=1 / c+b / c^{2}$ for $c \neq 0$ and $c, b \in R$. Let $w_{0, n}$ be defined recursively by

$$
-w_{0, n}-b w_{0, n+1}+c w_{0, n+2}=1, n=0,1,2, \ldots
$$

and let

$$
w_{k, n}=\sum_{i=0}^{n} w_{k-1, i}, \quad k=1,2, \ldots
$$

Then

$$
-w_{k, n}-b w_{k, n+1}+c w_{k, n+2}=\left(\begin{array}{c}
n+2+k \\
k
\end{array}\right) .
$$


This result first appeared in [5] and later in [6]. The generating function $g(x)$ for $(2.3)$ is given by

Theorem 2. Let $w_{k, n}$ be as in (2.3). Then the generating function $g(x)$ is given by

$$
g(x)=\sum_{n=0}^{\infty} w_{k, n} x^{n}=\frac{1}{\left(c-b x-x^{2}\right)(1-x)^{k+1}}, k=0,1,2, \ldots .
$$

Proof. Let

$$
S(x)=\sum_{n=0}^{\infty} w_{k, n} x^{n} .
$$

After employing (2.5) in (2.3), multiplying by $x^{n}$ and summing, we obtain

$$
-\frac{c}{x^{2}}\left(w_{k, 0}+w_{k, 1} x\right)+\frac{c}{x^{2}} S(x)+\frac{b}{x} w_{k, 0}-\frac{b}{x} S(x)-S(x)=\sum_{n=0}^{\infty}\left(\begin{array}{c}
n+k+2 \\
k
\end{array}\right) x^{n}
$$

or,

$$
S(x)\left(\frac{c}{x^{2}}-\frac{b}{x}-1\right)=\frac{c}{x^{2}}\left(\frac{1}{c}+\left(\frac{k+1}{c}+\frac{b}{c^{2}}\right) x\right)-\frac{b}{c x}+\sum_{n=0}^{\infty}\left(\begin{array}{c}
n+k+2 \\
k
\end{array}\right) x^{n}
$$

or,

$$
S(x)\left(c-b x-x^{2}\right)=1+(k+1) x+\sum_{n=0}^{\infty}\left(\begin{array}{c}
n+k+2 \\
k
\end{array}\right) x^{n+2}=\frac{1}{(1+x)^{k+1}} .
$$

from which the result follows.

Consider now the polynomials $p_{1}(x)=-x^{2}-b x+c$ and $p_{2}(x)=c x^{2}-$ $b x-1$. $p_{2}(x)$ is the characteristic polynomial of $(2.3)$. The zeros of $p_{1}(x), p_{2}(x)$ and the relations among them reveal some interesting facts. Let the zeors of $p_{1}(x)$ be $\beta_{1}$ and $\beta_{2}$ that of $p_{2}(x)$ be $\alpha_{1}$ and $\alpha_{2}$. Then,

$$
\begin{gathered}
\beta_{1}=\frac{-b+\sqrt{b^{2}+4 c}}{2}, \beta_{2}=\frac{-b-\sqrt{b^{2}+4 c}}{2}, p_{1}(x)=-\left(x-\beta_{1}\right)\left(x-\beta_{2}\right) . \\
\alpha_{1}=\frac{b+\sqrt{b^{2}+4 c}}{2 c}, \alpha_{2}=\frac{b-\sqrt{b^{2}+4 c}}{2 c}, p_{2}(x)=c\left(x-\alpha_{1}\right)\left(x-\alpha_{2}\right) . \\
\frac{1}{p_{1}(x)}=\frac{1}{-\left(x-\beta_{1}\right)\left(x-\beta_{2}\right)}=\frac{1}{\beta_{2}-\beta_{1}}\left[\frac{1}{x-\beta_{1}}-\frac{1}{x-\beta_{2}}\right],
\end{gathered}
$$


and

$$
\frac{1}{p_{2}(x)}=\frac{1}{c\left(x-\alpha_{1}\right)\left(x-\alpha_{2}\right)}=\frac{1}{c\left(\alpha_{2}-\alpha_{1}\right)}\left[\frac{1}{x-\alpha_{2}}-\frac{1}{x-\alpha_{1}}\right] .
$$

This leads then to

\section{Proposition 1.}

$$
\frac{x}{p_{1}(x)}=\frac{1}{c\left(\alpha_{1}-\alpha_{2}\right)}\left[\frac{1}{1-x \alpha_{1}}-\frac{1}{1-x \alpha_{2}}\right]=\frac{1}{c} \sum_{n=0}^{\infty} x^{n} \frac{\alpha_{1}^{n}-\alpha_{2}^{n}}{\alpha_{1}-\alpha_{2}} .
$$

Proof. The proof follows from (2.6-2.9).

Using Proposition 1 and Theorem 2 we get,

\section{Proposition 2.}

$$
g(x)=\frac{1}{c} \sum_{n=1}^{\infty} \frac{\alpha_{1}^{n}-\alpha_{2}^{n}}{\alpha_{1}-\alpha_{2}} \frac{x^{n-1}}{(1-x)^{k+1}} .
$$

Proof. In Proposition 1, divide by $x$, sum from $n=1$ and use the definiton of $p_{1}(x)$.

From Theorem 1 and Proposition 2 it follows that,

Corollary 1. Let $a=b=1$ in Theorem 1. Define $w_{k, n}=w_{k, n}(1,1)$, then

$$
\sum_{n=0}^{\infty} w_{k, n}(1,1) x^{n}=\sum_{n=1}^{\infty} F_{n} \frac{x^{n-1}}{(1-x)^{k+1}} .
$$

Proof. This follows from Propostion 1 and the definition of the $n$-th Fibonacci number $F_{n}=\left(\alpha_{1}^{n}-\alpha_{2}^{n}\right) /\left(\alpha_{1}-\alpha_{2}\right)$, where $\alpha_{1}=(1+\sqrt{5}) / 2, \alpha_{2}=$ $(1-\sqrt{5}) / 2$.

Next, a closed form for $w_{k, n}$ is found using two approaches: analytic and inductive approach.

Theorem 3.

$$
\begin{aligned}
w_{k, n} & =\frac{1}{c} \sum_{j=0}^{n} \sum_{j-2 s \geq 0}\left(\begin{array}{c}
j-s \\
s
\end{array}\right) \frac{b^{j-2 s}}{c^{j-s}}\left(\begin{array}{c}
k+n-j \\
n-j
\end{array}\right) \\
& =\frac{1}{c} \sum_{j=0}^{n} \sum_{s=0}^{\lfloor j / 2\rfloor}\left(\begin{array}{c}
j-s \\
s
\end{array}\right) \frac{b^{j-2 s}}{c^{j-s}}\left(\begin{array}{c}
k+n-j \\
k
\end{array}\right) .
\end{aligned}
$$


where $\lfloor j / 2\rfloor$ denotes the 'floor' of $j / 2$.

Proof. Analytic Proof.

$$
\frac{1}{(1-x)^{k+1}}=1+\left(\begin{array}{c}
k+1 \\
1
\end{array}\right) x+\left(\begin{array}{c}
k+2 \\
2
\end{array}\right) x^{2}+\ldots+\left(\begin{array}{c}
k+n \\
k
\end{array}\right) x^{n}+\ldots
$$

Using (2.12), the coefficient of $x^{n}$ in Propostion 2 is,

$$
\sum_{j=0}^{n}\left(\begin{array}{c}
k+n-j \\
k
\end{array}\right) \frac{\alpha_{1}^{j+1}-\alpha_{2}^{j+1}}{\alpha_{1}-\alpha_{2}}
$$

The result follows by expanding $\left(\alpha_{1}^{j+1}-\alpha_{2}^{j+1}\right) /\left(\alpha_{1}-\alpha_{2}\right)$.

Proof. Inductiive Proof. Solving for $w_{k, n+2}$ from (2.3) yields,

$$
w_{k, n+2}=\frac{1}{c}\left(\begin{array}{c}
k+n+2 \\
k
\end{array}\right)+\frac{b}{c} w_{k, n+1}+\frac{1}{c} w_{k, n} .
$$

The conditions,

$$
w_{k, 0}=\frac{1}{c}
$$

and

$$
w_{k, 1}=\frac{1}{c}\left[\left(\begin{array}{c}
k+1 \\
1
\end{array}\right)+\frac{b}{c}\right]
$$

follow from (2.2).

Taking $n=0$ in (2.13) and employing (2.14), (2.15) one obtains,

$$
w_{k, 2}=\frac{1}{c}\left[\left(\begin{array}{c}
k+2 \\
2
\end{array}\right)+\frac{b}{c}\left(\begin{array}{c}
k+1 \\
1
\end{array}\right)+\left(\frac{b^{2}}{c^{2}}+\frac{1}{c}\right)\right] .
$$

Similarly,

$$
\begin{array}{r}
w_{k, 3}=\frac{1}{c}\left[\left(\begin{array}{c}
k+3 \\
3
\end{array}\right)+\frac{b}{c}\left(\begin{array}{c}
k+2 \\
2
\end{array}\right)+\left(\frac{b^{2}}{c^{2}}+\left(\begin{array}{l}
1 \\
1
\end{array}\right) \frac{1}{c}\right)\left(\begin{array}{c}
k+1 \\
1
\end{array}\right)\right. \\
\left.+\left(\frac{b^{3}}{c^{3}}+\left(\begin{array}{l}
2 \\
1
\end{array}\right) \frac{1}{c} \cdot \frac{b}{c}\right)\right] .
\end{array}
$$

Inspection of (2.16),(2.17) (eventually) yields a pattern. By induction, assume for all $v \leq n$,

$$
w_{k, v}=\frac{1}{c} \sum_{j=0}^{v} \sum_{j-2 s \geq 0}\left(\begin{array}{c}
j-s \\
s
\end{array}\right) \frac{b^{j-2 s}}{c^{j-2 s}} \cdot \frac{1}{c^{s}}\left(\begin{array}{c}
k+v-j \\
k
\end{array}\right)
$$


Replacing $v$ by $n+1, n \geq 1$ in (2.18) and employing (2.3) gives,

$$
\begin{aligned}
w_{k, n+1} & =\frac{1}{c}\left(\begin{array}{c}
k+n+1 \\
k
\end{array}\right)+\frac{b}{c} w_{k, n}+\frac{1}{c} w_{k, n-1}+ \\
& =\frac{1}{c}\left(\begin{array}{c}
k+m+1 \\
k
\end{array}\right)+\frac{1}{c} \cdot \frac{b}{c}\left(\begin{array}{c}
k+n \\
k
\end{array}\right) \\
& +\frac{1}{c} \sum_{j=1}^{n} \sum_{j-2 s \geq 0}\left(\begin{array}{c}
j-s \\
s
\end{array}\right) \frac{b^{j+1-2 s}}{c^{j+1-2 s}} \cdot \frac{1}{c^{s}}\left(\begin{array}{c}
k+n-j \\
k
\end{array}\right) \\
& +\frac{1}{c} \sum_{j=0}^{n-1} \sum_{j-2 s \geq 0}\left(\begin{array}{c}
j-s \\
s
\end{array}\right) \frac{b^{j-2 s}}{c^{j-2 s}} \cdot \frac{1}{c^{s+1}}\left(\begin{array}{c}
k+n-1-j \\
k
\end{array}\right) \\
& =\frac{1}{c}\left(\begin{array}{c}
k+n+1 \\
k
\end{array}\right)+\frac{1}{c}\left(\begin{array}{c}
k+n \\
c
\end{array}\right) \\
& +\frac{1}{c} \sum_{j=0}^{n-1} \sum_{j+1-2 s \geq 0}\left(\begin{array}{c}
j+1-s \\
s
\end{array}\right) \frac{b^{j+2-2 s}}{c^{j+2-2 s}} \cdot \frac{1}{c^{s}}\left(\begin{array}{c}
k+n-j-1 \\
k
\end{array}\right) \\
& +\frac{1}{c} \sum_{j=0}^{n-1} \sum_{j+2-2 s \geq 0}\left(\begin{array}{c}
j+1-s \\
s-1
\end{array}\right) \frac{b^{j+2-2 s}}{c^{j+2-2 s}} \cdot \frac{1}{c^{s}}\left(\begin{array}{c}
k+n-1-j \\
k
\end{array}\right) .
\end{aligned}
$$

Note that in the last term $s \geq 1$. The useful identity,

$$
\left(\begin{array}{c}
j+1-s \\
s
\end{array}\right)+\left(\begin{array}{c}
j+1-s \\
s-1
\end{array}\right)=\left(\begin{array}{c}
j+2-s \\
s
\end{array}\right),
$$

can simplify equation (2.19) to obtain,

$$
\begin{aligned}
w_{k, m+1} & =\frac{1}{c}\left(\begin{array}{c}
k+n+1 \\
k
\end{array}\right)+\frac{b}{c} \cdot \frac{1}{c}\left(\begin{array}{c}
k+n \\
k
\end{array}\right) \\
& +\frac{1}{c} \sum_{j=0}^{n-1} \sum_{j+2-2 s \geq 0}\left(\begin{array}{c}
j+2-s \\
s
\end{array}\right) \frac{b^{j+2-2 s}}{c^{j+2-2 s}} \cdot \frac{1}{c^{s}}\left(\begin{array}{c}
k+n-j-1 \\
k
\end{array}\right) \\
& =\frac{1}{c}\left(\begin{array}{c}
k+n+1 \\
k
\end{array}\right)+\frac{b}{c} \cdot \frac{1}{c}\left(\begin{array}{c}
k+n \\
k
\end{array}\right) \\
& +\frac{1}{c} \sum_{j=2}^{n+1} \sum_{j-2 s \geq 0}\left(\begin{array}{c}
j-s \\
s
\end{array}\right) \frac{b^{j-2 s}}{c^{j-2 s}} \cdot \frac{1}{c^{s}}\left(\begin{array}{c}
k+n+1-j \\
k
\end{array}\right) .
\end{aligned}
$$


Combining terms in (2.20) gives

$$
w_{k, n+1}=\frac{1}{c} \sum_{j=0}^{n+1} \sum_{s=0}^{\lfloor j / 2\rfloor}\left(\begin{array}{c}
j-s \\
s
\end{array}\right) \frac{b^{j-2 s}}{c^{j-s}}\left(\begin{array}{c}
k+n+1-j \\
k
\end{array}\right) .
$$

The proof is complete by noting that when $j$ is odd, $s=0,1, \ldots, j-1$.

From Theorem 3 one gets

\section{Corollary 2.}

$$
w_{k, n}=w_{k, n}(1,1)=\sum_{j=0}^{n} F_{j+1}\left(\begin{array}{c}
k+n-j \\
k
\end{array}\right) .
$$

With $w_{k, n}$ given by 2.11 and employing Proposition 2 it follows that,

\section{Theorem 4.}

$$
\frac{\alpha_{1}^{n+1}-\alpha_{2}^{n+1}}{\alpha_{1}-\alpha_{2}}= \begin{cases}\sum_{i=0}^{k+1} w_{k, n-i}(-1)^{i}\left(\begin{array}{c}
k+1 \\
i
\end{array}\right), & \text { if } n \geq k+1 \\
\sum_{i=0}^{n} w_{k, n-i}(-1)^{i}\left(\begin{array}{c}
k+1 \\
i
\end{array}\right), & \text { if } n \leq k .\end{cases}
$$

Proof. In Proposition 2, multiply both sides by $(1-x)^{k+1}$, use definition of $g(x)$ and expand to find power of $x^{n}$.

\section{Corollary 3.}

$$
F_{n+1}= \begin{cases}\sum_{i=0}^{k+1} w_{k, n-i}(1,1)(-1)^{i}\left(\begin{array}{c}
k+1 \\
i
\end{array}\right), & \text { if } n \geq k+1 ; \\
\sum_{i=0}^{n} w_{k, n-i}(1,1)(-1)^{i}\left(\begin{array}{c}
k+1 \\
i
\end{array}\right), & \text { if } n \leq k .\end{cases}
$$

The case $b=0$ is also considered to get the following,

Corollary 4. If $b=0$ in Theorem 2, then

$$
a_{k, n}=\frac{1}{c} \sum_{s=0}^{\lfloor n / 2\rfloor} \frac{1}{c^{s}}\left(\begin{array}{c}
k+n-2 s \\
k
\end{array}\right) .
$$

Proof. The result follows directly from (2.11).

Several examples involving the golden section, the Fibonacci and the Lucas numbers are presented in next section. 


\section{Combinatorial Identities}

In this section we present some examples for the representation of a linear combination of the Fibonacci and Lucas numbers that involve a polynomial and a double sum combinatorial term. These representations are derived by employing the method of undetermined coefficients for specific values of $k$ in (2.3).

Case 1: $\mathrm{k}=0$.

$$
a_{1, n}=D+c_{1} \alpha^{n}+c_{2} \beta^{n},
$$

for some real constants $D, c_{1}, c_{2}$. This yields, upon substitution into (2.3),

$$
-D-b D+c D=\left(\begin{array}{l}
n+2 \\
n+2
\end{array}\right)
$$

which yields,

$$
D=-\frac{1}{b+1-c}
$$

By solving the characteristic equation of (2.3),

$$
-1-b x+c x^{2}=0
$$

we get

$$
\alpha=\frac{b+\sqrt{b^{2}+4 c}}{2 c}, \beta=\frac{b-\sqrt{b^{2}+4 c}}{2 c} .
$$

Let $b=c=1$. This gives, $\alpha=\frac{1+\sqrt{5}}{2}, \beta=\frac{1-\sqrt{5}}{2}$. Employing (3.5), (3.3), in (3.1) and using the initial conditions for $n=0$, 1 , yields

$$
D=-1, c_{1}=\frac{2 \sqrt{5}}{5}+1, c_{2}=-\frac{2 \sqrt{5}}{5}+1 .
$$

Employing (3.6) in (3.1), and equating it to (2.11) yields

$$
\begin{aligned}
& \left(\frac{2 \sqrt{5}}{5}+1\right)\left(\frac{1+\sqrt{5}}{2}\right)^{n}+\left(\frac{-2 \sqrt{5}}{5}+1\right)\left(\frac{1-\sqrt{5}}{2}\right)^{n} \\
& =2 F_{n}+L_{n}=\frac{4}{2} F_{n}+\frac{2}{2} L_{n}=\frac{1}{2}\left(L_{3} F_{n}+F_{3} L_{n}\right) \\
& =1+\sum_{j=0}^{n} \sum_{s=0}^{\lfloor j / 2\rfloor}\left(\begin{array}{c}
j-s \\
s
\end{array}\right)=1+\sum_{j=0}^{n} F_{j+1} .
\end{aligned}
$$


which is known already.

Case 2: $\mathrm{k}=1$

$$
\begin{aligned}
& \left(\frac{11 \sqrt{5}}{10}+\frac{5}{2}\right)\left(\frac{1+\sqrt{5}}{2}\right)^{n}+\left(\frac{-11 \sqrt{5}}{10}+\frac{5}{2}\right)\left(\frac{1-\sqrt{5}}{2}\right)^{n} \\
& =\frac{11}{2} F_{n}+\frac{5}{2} L_{n}=\frac{1}{2}\left(L_{5} F_{n}+F_{5} L_{n}\right) \\
& =n+4+\sum_{j=0}^{n} \sum_{s=0}^{\lfloor j / 2\rfloor}\left(\begin{array}{c}
j-s \\
s
\end{array}\right)\left(\begin{array}{c}
n-j+1 \\
n-j
\end{array}\right),
\end{aligned}
$$

\section{References}

[1] Mohammad Azarian, Identities Involving Lucas or Fibonacci and Lucas Numbers as Binomial Sums, Int. J. Contem. Math. Sciences, 7:45(2012), 2221-2227.

[2] David M. Burton, The History of Mathematics, An Introduction, McGraw Hill, 7th Ed., 2011.

[3] Henry W. Gould, Combinatorial identities. A standardized set of tables listing 500 binomial coefficient summations Henry W. Gould, Morgantown, W.Va., 1972.

[4] Ronald L. Graham, Donald E. knuth, Oren Patashnik, Concrete Mathematics, A Foundation for Computer Science, Addison-Wesley, 1989.

[5] George Grossman, Aklilu Zeleke, On linear recurrence relations and combinatorial identities, Journal of Concrete and Applicable Mathematics, Vol. 1, No. 3, pp. 229-245, Nova Science Publishers, 2003.

[6] George Grossman, Linear recurrence relations and the binomial coefficients, in Proceedings of XII ${ }^{\text {th }}$ CZECH-POLISH-SLOVAK Mathematical School by the Faculty of Education of University J. E. Purkynĕ, Ústí nad Labem, Hublos̆, June 2-4, 2005, 111-119.

[7] H. H. Gulec, N. Taskara, On the Properties of Fibonacci Numbers with Binomial Coefficients, Int. J. Contemp. Math. Sciences,4, No. 25, (2009),1251-1256.

[8] Thomas Koshy, Fibonacci Numbers and Lucas numbers with applications, John Wiley \& Sons, Inc., 2001

[9 ] George Pólya, Gabor Szegö, Aufgaben and Lehrsätze, New York, Dover Publications, 1954.

[10] John Riordan. Combinatorial Identities, John Wiley \& Sons Inc., 1968.

[11] Herbert S. Wilf, generatingufnctionology, Academic Press, Inc., 1994. 